\title{
Obstetrics and Gynecology Patients Postoperative Pain Effect Assessment by Comprehensive Nursing Intervention
}

\author{
Zhou Yan \\ (Shandong heze medical college, Shandong heze,274000)
}

\begin{abstract}
Keywords: Comprehensive nursing intervention; Obstetrics and gynecology department; Postoperative pain; Effect
\end{abstract}

\begin{abstract}
This paper evaluates and analyzes the effect of comprehensive nursing intervention on obstetrics and gynecology patients' postoperative pain. Methods: choose 214 obstetrics and gynecology patients with postoperative pain, and divide them into control group and observation group by hospital order, with 107 cases in each group. The control group is treated by routine nursing, and the observation group adopts comprehensive nursing intervention based on conventional nursing. Comparative analyze the average length of hospital stay, nursing service satisfaction and degree of pain of the two groups of patients. Results: after $2 \mathrm{~d}, 4 \mathrm{~d}$ and $6 \mathrm{~d}$ of the implementation of comprehensive nursing intervention, compared with control group, the pain score status of observation group patients are significantly lower $(\mathrm{P}<0.05)$; Observation group patients' average length of hospital stay and nursing service satisfaction, compared with the control group, is superior $(\mathrm{P}<0.05)$, this comparing difference has statistical significance. Conclusion: for obstetrics and gynecology patients with postoperative pain, the implementation of comprehensive nursing intervention has remarkable nursing effect. Patients have obvious pain relief, and it also effectively shortens hospitalization time and improves patients' satisfaction of nursing service. It has good clinical popularization value.

In clinical obstetrics and gynecology, the most common method of treatment in obstetrics and gynecology operating room is adopted for patients with gynecological disease and abnormal childbirth puerpera, and when the patients' local tissue integrity is damaged, patients feel pain obviously. In postoperative pain, patient's body is long in the stress state and easy to cause bad feelings and negative effects on incision healing, more severe cases can cause corresponding post-operational complications. Relevant clinical practice has proved that the implementation of comprehensive nursing intervention for obstetrics and gynecology patients with postoperative pain has ideal clinical therapeutic effects [1].
\end{abstract}

\section{DATA AND METHODS}

\section{A. General information}

Select 214 cases of obstetrics and gynecology patients with postoperative pain with age range of $27 \sim 60$ years old, and the average age is $(33.4 \pm 2.6)$. The plane palace production operation is 124 cases, 20 patients with hysterectomy, 37 appendix removed patients, 33 patients with pelvic cleaning operation, and all the patients have finished surgery. The selected patients have no hepatic and renal complications, no corresponding operation contraindication, no allergic history of drug abuse, and are volunteers in the study. They are divided into control group and observation group according to hospitalized order, and each group has 107 cases. There is age difference between two patients groups, and no statistical significance $(\mathrm{P}>0.05)$.

\section{B. Nursing method}

Control group: control group patients are treated by routine nursing with preoperative routine body inspection, and are asked to pay more attention to daily rest. Implement effective monitoring on postoperative heart rate, pulse and blood pressure, etc. of the patients, and guide patients in drug and food, etc. Observation groups: observation group patients get comprehensive nursing intervention on the conventional nursing foundation, and the nursing measures are as follows: (1) Preoperative health education support. Explain postoperative pain knowledge content to the preoperative patients, also inform the patients' postoperative pain relief method to help eliminate 
the fear effect of postoperative pain, and improve patients' psychological tolerance for pain [2]. (2) Psychological nursing. Obstetrics and gynecology surgery will cause great physical and mental influence on women. Some women can produce different levels of anxiety and fear because of the remove of uterus or attachments, and the related nursing staff should actively give spiritual communication and encouragement to patients and their families, and nurses' attitudes should keep warm and kind to eliminate strangeness existing between nurses and patients. According to patient's anxiety and fear, nursing staff should timely provide psychological counsel to patients for operation, and patients' families should strengthen the care of patients, try our best to help patients to build confidence and courage to conquer the disease [3]. (3) Diet nursing care. Patients should be advised to eat more delicate food. After the patient can have normal diet activity, nursing staff should advise patients to eat more foods containing high cellulose, and to ensure patient air-frame nutrition in balance. (4) Pain nursing. Postoperative patients comfortable positions should avoid wound oppression to prevent the occurrence of cracking in patients wound condition, leading to worse pain. For patients with cesarean section, when use analgesia pump, nursing staff should give close observation of their vital signs, take corresponding nursing measures analgesia pump; when maternal body posture change, protect catheter and prevent catheter snap and reflect phenomenon [4]. (5) Body relaxation training. Play light music inside the patients room, make positive guidance for the body to relax, urge patients to take a deep breath, achieve the goal of muscles relax, guide the patients to keep peace and happy mentality, and reach the purpose of anxiety in patients with ease. The training should last $1 \mathrm{~d} / \mathrm{a}, 1$ times / $35 \mathrm{~min}$. (5) other nursing care. Postoperative wound care is very important, because most of the patients' pain occur in the wound site. Every day clean up the wound and replace drug and excipients on time, mainly observe window form, whether any swelling, exudate, and so on and so forth, take emergency measures for the wound, pay attention to keep the wound clean, at the same time give anti-infection treatment, and prevent pain of wound infection; pay attention to the postoperative patient's diet instruction and avoid eating spicy cold hard and excitant food, eat more rich protein, vitamins and calcium food, and give priority to delicate food. Patients with a longer duration or postoperative pain need auxiliary analgesics, and should strictly take prescribed medication under doctors' supervision. No long-term uses avoid drug dependence.

\section{Observation indexes and evaluation methods}

According to world health organization (who) criteria evaluation, evaluate pain in patients, divide into four levels: level 0 is painless; level I is mild pain, patients can endure pain, and work normally; basically it will not affect the rest; level II is moderate pain, unbearable pain, which affects sleep, and need to be given a sedative; level III is severe pain, persistent pain and cannot bear with serious influence on sleep, need to give analgesic drugs, at the same time use our nursing department designed nursing job satisfaction questionnaire to survey patients' nursing staff and nurses job satisfaction, which is divided into 4 levels: satisfied, more satisfied, generally satisfied and unsatisfied, and satisfaction $=$ satisfaction rate + more satisfaction rate.

\section{Statistical methods}

According to the institute used data, use statistical software SPSS18.0 for analysis, use the standard deviation $(x \pm s)$ for measurement data, use $\mathrm{t}$ and $\mathrm{x} 2$ to test and count data respectively, the differences between two groups are denoted by $\mathrm{P}<0.05$.

\section{RESULTS}

\section{A. Comparison analysis of two groups patients pain score before and after treatment}

Implement corresponding nursing measures to two groups patients on preoperative and postoperative days, and pain score difference is not obvious, so the situation has no statistical significance ( $\mathrm{P}>0.05$ ); on the $2 \mathrm{~d}, 4 \mathrm{~d}$ and $6 \mathrm{~d}$ after implementation of comprehensive nursing intervention, compared with control group, pain score status of the observation group patients is significantly lower than the latter $(\mathrm{P}<0.05)$. See Table 1. 
Table 1. Two groups of patients pain score comparison before and after treatment $\left({ }^{x} \pm s\right)$

\begin{tabular}{llllll}
\hline Group & $\begin{array}{l}\text { Before } \\
\text { treatment }\end{array}$ & Day 1 & Day 2 & Day4 & Day 6 \\
\hline $\begin{array}{l}\text { Observation } \\
\text { group }\end{array}$ & $7.85 \pm 1.32$ & $7.23 \pm 1.01$ & $5.22 \pm 1.05$ & $4.12 \pm 1.16$ & $2.77 \pm 0.46$ \\
$\begin{array}{l}\text { Control } \\
\text { group }\end{array}$ & $7.88 \pm 1.29$ & $7.15 \pm 0.88$ & $6.89 \pm 1.17$ & $5.45 \pm 1.12$ & $4.79 \pm 1.02$ \\
$\mathrm{t}$ & 1.651 & 1.214 & 3.219 & 3.224 & 3.016 \\
\hline $\mathrm{P}$ & $>0.05$ & $>0.05$ & $<0.05$ & $<0.05$ & $<0.05$ \\
\hline
\end{tabular}

B. Two groups patients' average length of hospital stay and comparative analysis on nursing satisfaction score

The average length of hospital stay of the observation group, compared with control group, is superior $(\mathrm{P}<0.05)$, and patients nursing service satisfaction is superior $(\mathrm{P}<0.05)$, which is shown in Table 2.

Table 2 Two groups patients average length of hospital stay and comparison on nursing satisfaction

\begin{tabular}{cccc}
\multicolumn{4}{c}{ score $(\bar{x} \pm \mathrm{s})$} \\
\hline Group & Cases & $\begin{array}{c}\text { Average } \\
\text { hospitalization time }\end{array}$ & $\begin{array}{c}\text { Nursing } \\
\text { satisfaction }\end{array}$ \\
\hline Observation group & 38 & $8.2 \pm 2.1$ & $97.2 \pm 1.4$ \\
\hline Control group & 38 & $12.1 \pm 2.0$ & $86.4 \pm 2.1$ \\
\hline
\end{tabular}

\section{DISCUSSION}

Since most women's hearts are fragile, the tolerance to pain is not high. After implemented obstetrics and gynecology surgery, pain occurs in the situation, which will cause the patients fear, anxiety and other adverse psychological status, and cause certain influence on body's effective recovery. So for obstetrics and gynecology patients with postoperative pain, deal with effective comprehensive nursing intervention measures to help patients relieve their inner pain, encourage patients to early realize rehabilitation hospital discharge [5]. By preoperative health education, deepen patients' understanding of their disease, strengthen postoperative pain tolerance, and help to reduce negative effects of operation and treatment effect. Effective psychological nursing can improve the psychological status of patients, reduce negative psychological pain, and increase patients' pain tolerance threshold. In addition, by playing music and a variety of forms communication, calm the nerves of patients, and diversify their attention to effectively relieve the pain. Through this study, after the implementation of conventional nursing and comprehensive nursing intervention, two groups' patients' pain scores fall. Especially on Day2, Day4, and Day6, the observation group patients pain score status, compared with control group, is significantly lower $(\mathrm{P}<0.05)$; And on Day4, the control group get effective improvement in pain score, which shows that the conventional nursing methods also have a certain effect of care for gynecology and obstetrics patients with postoperative pain, but in concrete work, it is too slow, and the patients have to deal with pain longer. The average length of hospital stay and nursing service satisfaction of observation group patients, compared with control group, is superior, therefore, in view of obstetrics and gynecology patients with postoperative pain, adopt comprehensive nursing intervention measures, and the patient pain relief situation is more apparent.

To sum up, in view of gynecology and obstetrics patients with postoperative pain, the implementation of comprehensive nursing intervention has remarkable nursing effect, and patients get obvious pain relief, and it also effectively shortens hospitalization time and improves patients' satisfaction of nursing service, so it has good clinical popularization value. 


\section{REFERENCES}

[1] Feng Zhijuan. Postoperative patients pain maternity line comprehensive nursing intervention analysis [J]. Journal of Jilin Medical, 2014:172-172.

[2] Mao, Xiao Run, d. U. Wen Juan, and x. U. Make - Zhen. "Comprehensive Nursing Intervention for Prevention of Accidental Falls of the Community Elderly Patients with Hypertension or Diabetes," the Chinese General Practice16 (2013) : 575-578.

[3] Tang Xianju. Nursing intervention comprehensive nursing experience effect on postoperative pain of obstetrics and gynecology patients[J]. The world's Latest Medical Information Excerpt: Digital, 2013:479-479.

[4] Ao Lifen. Obstetrics and gynecology patients postoperative pain comprehensive nursing intervention analysis [J]. Journal of Grassroots Medical BBS, 2014:3622-3623.

[5] Nong Aili. Comprehensive nursing intervention impact on postoperative pain of obstetrics and gynecology patients and satisfaction [J]. Journal of Jilin Medical, 2014:6178-6179.

[6] Fang Yazhen. Nursing intervention impact on obstetrics and gynecology patients pain after surgery and quality of life [J]. Chinese Journal of Practical Nursing, 2011, the Roman - 28.

[7] Qian Xiaoya. Postoperative patients pain comprehensive nursing intervention effect evaluation [J]. Chinese Journal of Practical Nursing, 2012, 28 (6) : 41 and 42.

[8] Wei Xiuqin. The effect evaluation of comprehensive nursing intervention on postoperative pain of obstetrics and gynecology patients [J]. Journal of Modern Diagnosis and Treatment, 2013, $11: 2636-2636$. 